\title{
Utilisation de l'ICP-MS en biologie clinique
}

\section{Use of ICP-MS in clinical biology}

\section{Christian MCESCH*}

Faculté de Pharmacie et Service de Pharmacologie \& Toxicologie, Limoges

*Auteur à qui adresser la correspondance : Christian MCESCH, Faculté de Pharmacie de Limoges et Service de Pharmacologie \& Toxicologie, CHU Dupuytren, 2, avenue Martin Luther King, 87042 Limoges cedex

Tél : 0555056140 - Fax : 0555056162 - E-mail : christian.moesch@unilim.fr

(Reçu le 5 février 2007 ; accepté après modifications le 6 mars 2007)

\section{RÉSUMÉ}

L'analyse des éléments, métaux ou métalloïdes, dans diverses matrices n'a pas échappé au progrès scientifique et l'utilisation de la spectrométrie de masse inorganique couplée à un plasma induit par haute fréquence (ICP-MS) en est une parfaite illustration.

D'introduction relativement récente pour les analyses biologiques médicales, l'ICP-MS présente les avantages liés à l'association des deux techniques : le plasma couplé par induction apporte l'étendue de l'ensemble des éléments accessibles associée à une grande sensibilité et la spectrométrie de masse apporte également sa sensibilité (plus de soixante éléments ont leur limite de détection en dessous de $10 \mu \mathrm{g} / \mathrm{L}$ ), l'étendue de sa gamme dynamique linéaire s'étalant sur huit ordres de grandeur ainsi que la rapidité d'analyse. Une autre qualité de l'ICP-MS est son aptitude à la distinction et au dosage spécifique des isotopes d'un même élément, essentielle par exemple pour certaines études nutritionnelles. Bien évidemment, à la technique ICP-MS sont associés des problèmes spécifiques et des limitations comme celles liées aux effets physiques ou chimiques de matrice, tels que les interférences isobariques qu'il convient de supprimer.

D'exécution très rapide, cette méthode convient à des analyses en série ou en continu permettant le couplage avec la chromatographie liquide ou l'électrophorèse capillaire, ce qui la rend précieuse pour les analyses de spéciation et offre des perspectives nouvelles en biologie et toxicologie clinique.

\section{MOTS-CLÉS}

ICP-MS, biologie, métaux, métallö̈des.

\section{SUMMARY}

The analysis of the elements, metals or metalloids, in various matrices has benefited from scientific progress and the use of inorganic mass spectrometry coupled with plasma induced by high frequency (ICP-MS) is a perfect illustration.

ICP-MS, which has only fairly recently been introduced into medical biological analyses, has the advantages related to the association of the two techniques: the inductively coupled plasma gives the extent of the accessible elements associated with a high-level of sensibility whereas mass spectrometry brings also its sensibility (more than sixty elements have a detection limit below $10 \mu \mathrm{g} / \mathrm{L}$ ), the extent of its linear dynamic range covering eight orders of magnitude as well as the speed of analysis. Another quality of the ICP-MS is its aptitude for the distinction and the specific dosage of the isotopes of the same element which are essential, for example, for certain nutritional studies. Obviously, specific problems and limitations are associated with the ICP-MS technique like those related to the physical or chemical effects of matrix, such as the isobaric interferences which it is advisable to remove.

This method which has a very fast execution is appropriate for series of analyses or continuous ones, allowing the coupling with the liquid chromatography or the capillary electrophoresis. This makes it invaluable for the analyses of speciation and offers new prospects in biology and clinical toxicology.

\section{KEY-WORDS \\ ICP-MS, biology, metals, metalloids.}




\section{Introduction}

L'émergence de la spectrométrie de masse couplée à un plasma induit par haute fréquence (ICP-MS) dans les années quatre-vingt (1) a laissé entrevoir les possibilités énormes de cette technique dans des domaines aussi diversifiés que l'environnement, la géologie ou la microélectronique, rapidement suivis par la biologie clinique (2-5). Pour cette dernière, les principales caractéristiques et critères à prendre en compte dans le choix d'une technique d'analyse des éléments traces sont rassemblés dans le tableau I, pour autant que l'on puisse donner un tableau général qui ne peut prendre en compte tous les cas particuliers liés à la spécificité d'un élément ou d'une matrice biologique. Il n'en demeure pas moins qu'en dehors d'un aspect financier, légèrement estompé par la commercialisation relativement récente d'appareils compacts dits de « paillasse », l'ICP-MS est la seule technique associant à l'information isotopique les deux atouts majeurs que sont la capacité multiélémentaire et la sensibilité.

Cette méthode instrumentale d'analyse qualitative et quantitative repose sur la séparation, l'identification et la quantification des éléments constitutifs d'un échantillon en fonction du rapport de leur masse $m$ à leur charge $z$. Elle associe donc à un spectromètre de masse (MS) une source d'ions formée d'une torche à plasma entretenu par couplage inductif avec un générateur haute fréquence (ICP). Elle présente par rapport aux techniques classiquement utilisées pour l'analyse inorganique des liquides biologiques, l'avantage des qualités du plasma induit par haute fréquence (potentiel multiélémentaire, très grande sensibilité, rapidité de succession possible des échantillons), associées à celles de la spectrométrie de masse (abaissement des seuils de détection, étendue de la gamme dynamique, rapidité d'analyse, possibilité de mesures isotopiques des éléments trace).

Après avoir présenté l'instrumentation et les problèmes spécifiques liés à la technique, l'article abordera l'utilisation en biologie clinique de l'ICP-MS : préparation des échantillons et exemples d'applications.

\section{Instrumentation problèmes spécifiques de l'ICP-MS (6-8)}

L'analyse des échantillons par ICP-MS peut être divisée en quatre étapes ayant chacune leurs problèmes spécifiques :

- introduction de l'échantillon et production de l'aérosol,

- ionisation à l'aide de la torche à plasma,

- séparation en masse,

- détection par comptage des ions à la masse considérée,

suivies du traitement informatique du signal.

\section{Introduction de l'échantillon et production de l'aérosol}

Dans le domaine des analyses biologiques médicales, la plupart des analyses réalisées par ICP-MS mettent en jeu des échantillons ayant été mis en solution. Bien qu'un plasma soit à une température suffisamment élevée $(6000$ à $8000 \mathrm{~K})$ pour pouvoir volatiliser n'importe quel type d'élément, le temps de séjour de l'échantillon dans le plasma est limité. Une bonne dissociation impose donc un faible apport de liquide sous forme d'un aérosol de taille micrométrique $(<5 \mu \mathrm{m})$. La production d'un tel aérosol peut être réalisée au moyen d'un nébuliseur pneumatique,

Tableau I : Principales caractéristiques des techniques d'analyse élémentaire utilisées en biologie clinique (SAA-F : spectrométrie d'absorption atomique avec atomisation par flamme ; SAA-ET : spectrométrie d'absorption atomique avec atomisation électrothermique ; ICP-AES : spectrométrie d'émission optique couplée à un plasma induit par haute fréquence ; ICP-MS : spectrométrie de masse couplée à un plasma induit par haute fréquence ; $L D D, L D Q$ : limites de détection, de quantification)

\begin{tabular}{|c|c|c|c|c|}
\hline & SAA-F & SAA-ET & ICP-AES & ICP-MS \\
\hline Nombre global d'éléments déterminables & $>68$ & $>50$ & $>75$ & $>75$ \\
\hline Nombre d'éléments avec une LDQ $<10 \mu \mathrm{g} / \mathrm{L}$ & 24 & 46 & 55 & $>60$ \\
\hline Sélectivité & ++++ & +++++ & +++ & +++ \\
\hline Gamme dynamique & $10^{2}-10^{3}$ & $10^{2}-10^{3}$ & $10^{5}-10^{6}$ & $10^{8}$ \\
\hline LDD usuelles en biologie clinique $(\mu \mathrm{g} / \mathrm{L})$ & $10-1000$ & $1-50$ & $5-50$ & $0,1-10$ \\
\hline Echantillon : concentration en sels dissous $(\mathrm{g} / \mathrm{L})$ & $0,5-3$ & $>30$ & $1-30$ & $0,1-0,4$ \\
\hline Echantillon : volume minimum nécessaire $(\mathrm{mL})$ & 0,2 & $0,1-0,2$ & $0,2-1$ & $0,2-0,5$ \\
\hline Cadence analytique & ++ & + & +++ & +++ \\
\hline Utilisation en routine & ++++ & ++ & +++ & ++ \\
\hline Investissement financier & x 0,5 & $\mathrm{x} 1$ & $\mathrm{x} 2$ & $\mathrm{x} 4$ \\
\hline
\end{tabular}


classiquement de type concentrique Meinhard $\AA$, associant sensibilité, précision et faible consommation de liquide (1 à $2 \mathrm{~mL} / \mathrm{min}$ en version standard). Par contre ses inconvénients sont la fragilité et la possibilité de bouchage du capillaire, l'une des raisons nécessitant de diluer les échantillons de façon à ce que la concentration en sels dissous soit réduite $(<1 \%)$. Dans ce type de nébuliseur, l'échantillon mobilisé par une pompe péristaltique est introduit dans un tube capillaire (diamètre $200 \mu \mathrm{m}$ ) autour duquel un flux d'argon circulant à vitesse sonique le nébulise. Le débit du gaz de nébulisation doit être optimisé de façon à obtenir une réponse maximale pour l'analyte, tout en tenant compte du fait que l'augmentation du débit diminue le temps de séjour de l'échantillon dans le plasma. Ceci entraîne une moindre ionisation se traduisant par une diminution du taux d'ions doublement chargés (rapport $\mathrm{Ba}^{++} / \mathrm{Ba}^{+}<3 \%$ ) mais aussi par une augmentation du taux d'oxydes (rapport $\mathrm{CeO}^{+} / \mathrm{Ce}^{+}<3 \%$ ) puisque leur dissociation est réduite. Il s'agit ici d'un paramètre critique dans l'optimisation quotidienne des performances de l'appareil pour un analyte donné comme le montre la figure 1 .

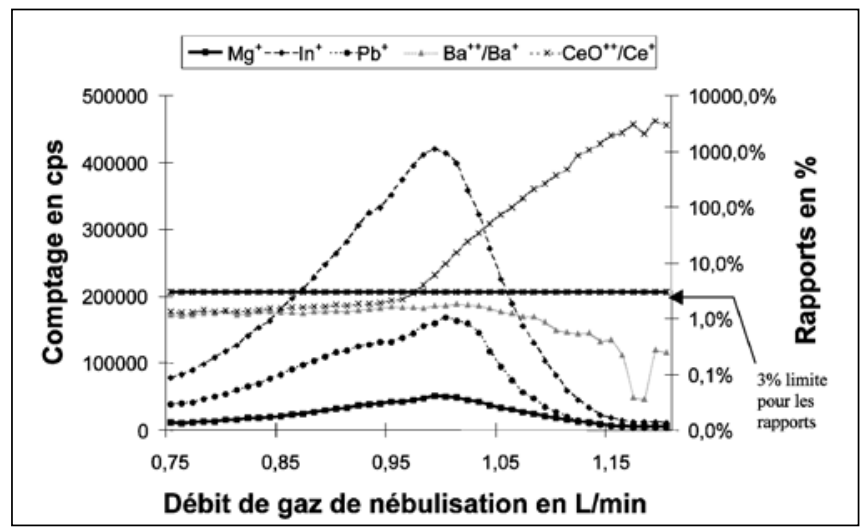

Figure 1 : Evolution des signaux de $\mathrm{Mg}^{+}, \mathrm{In}^{+}, \mathrm{Pb}^{+}$et des taux d'ions doublement chargés $\mathrm{Ba}^{++} / \mathrm{Ba}^{+}$et d'oxydes $\mathrm{CeO}^{+} / \mathrm{Ce}^{+}$en fonction du débit de gaz de nébulisation - ICP-MS Elan 6100 DRC - Perkin Elmer Sciex (10).

L'aérosol produit pénètre ensuite dans une chambre de nébulisation dont le rôle est de trier les gouttes selon leur taille et de ne transmettre au plasma que les plus petites (rendement de quelques \%), les plus grosses (> $10 \mu \mathrm{m}$ ), susceptibles de déstabiliser le plasma, partant au rejet. En ICP-MS, le modèle le plus souvent utilisé est la chambre cyclonique dont le faible volume mort diminue le temps de rinçage et augmente de fait la cadence analytique. Sur certains appareils, une réfrigération de la chambre de nébulisation (effet Peltier ou fluide réfrigérant) favorise la stabilisation de l'aérosol et permet de minimiser le taux d'oxydes par une réduction de la quantité de solvant envoyée vers le plasma.

\section{Ionisation à l'aide de la torche à plasma}

L'échantillon arrive alors dans une torche à plasma d'argon, à très haute température, suffisante pour atomiser et ioniser plus de 75 éléments ; alcalins, alcalino-terreux, métaux, métalloïdes et lanthanides sont ionisés à plus de $90 \%$ et seuls quelques éléments à haute énergie d'ionisation ne sont que partiellement ionisés : $\mathrm{H}, \mathrm{C}, \mathrm{N}, \mathrm{O}$, halogènes, soufre, mercure. Cette ionisation partielle peut néanmoins être suffisante pour quantifier le brome, l'iode ou le mercure dans les milieux biologiques. L'argon est généralement utilisé comme gaz plasmagène $(15 \mathrm{~L} / \mathrm{min})$ pour ses qualités : gaz rare (donc milieu chimiquement inerte et minimisant la formation possible d'ions polyatomiques), température élevée du plasma permettant une bonne efficacité d'atomisation, énergie d'ionisation élevée $(15,76 \mathrm{eV})$, supérieure au premier potentiel d'ionisation de la plupart des éléments de la classification périodique dont il est judicieux de connaitre les potentiels d'ionisation lors de la mise au point des méthodes de dosage (figure 2)

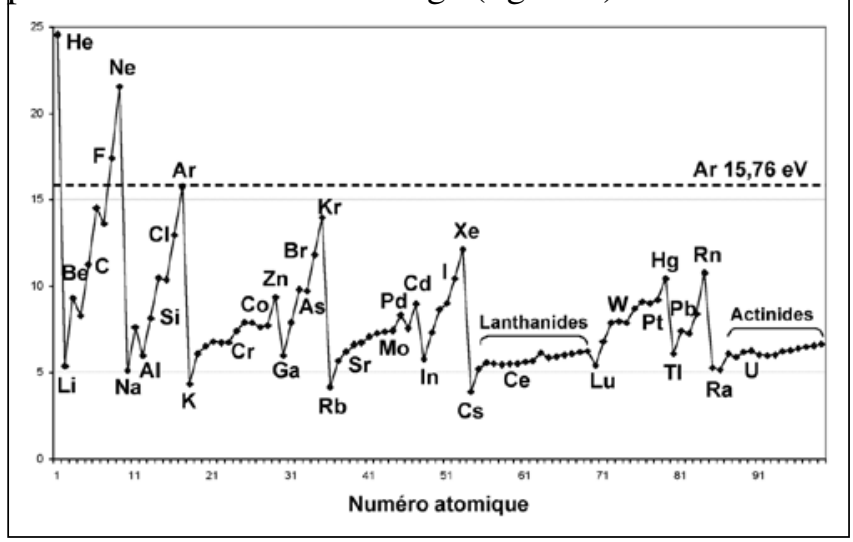

Figure 2 : Premiers potentiels d'ionisation des principaux éléments d'intérêt biologique en fonction de leur numéro atomique.

Un autre avantage de la torche à plasma est le confinement de l'échantillon dans la décharge et plus particulièrement dans un canal central produit par « effet de peau ». On a donc une zone de quelques millimètres de diamètre où vont se trouver situés les ions, ce qui facilite leur prélèvement. Par contre, la réalisation de l'interface entre la torche et le spectromètre est délicate, puisqu'il est nécessaire de prélever les ions au centre de la décharge $\left(6000 \mathrm{~K}, 10^{5} \mathrm{~Pa}\right)$ alors que le spectromètre de masse doit travailler à température ambiante et à très basse pression $\left(<10^{-3} \mathrm{~Pa}\right)$. Cette interface, utilisant la technique des jets supersoniques (Mach 5 à 7) associe successivement :

- un premier cône en nickel, échantillonneur ou «sampler », pour extraire les ions du plasma,

- un deuxième cône, écorceur ou « skimmer » percé d'un orifice de plus petit diamètre, pour prélever le centre 
du jet supersonique,

- une optique ionique constituée d'une ou plusieurs lentilles électrostatiques dont le rôle est de stopper les photons et de refocaliser le jet d'ions pour le rendre concentrique à l'axe du filtre quadripolaire.

Tous ces éléments nécessitent réglage et entretien régulier, notamment les cônes dont le nickel peut être source d'artefacts et dont les orifices peuvent s'éroder ou s'obstruer par des dépôts minéraux liés à la salinité des échantillons. Par ailleurs, l'optique ionique peut être précédée d'une chambre de collision ou d'une cellule de réaction dynamique (Dynamic Reaction Cell pour l'Elan 6100 DRC de Perkin Elmer Sciex) afin de dissocier certains ions polyatomiques provoquant des interférences isobariques (11) que l'on abordera plus loin.

\section{Séparation en masse et détection (figure 3)}

Les éléments ionisés sont amenés vers un analyseur de masse dont le rôle est de trier les ions selon leur rapport masse/charge $(\mathrm{m} / \mathrm{z})$. Il est caractérisé par son pouvoir de résolution, c'est-à-dire sa capacité à distinguer deux ions de rapports $\mathrm{m} / \mathrm{z}$ très proches. Les appareils classiquement utilisés en biologie clinique sont des spectromètres de masse quadripolaires, très sensibles et relativement économiques ; mais leur résolution, de l'ordre de 0,5 uma, est assez faible et peut être une limitation pour résoudre des interférences isobariques comme celle entre ${ }^{75} \mathrm{As}^{+}$et ${ }^{40} \mathrm{Ar}^{35} \mathrm{Cl}^{+}$. Ces interférences pourraient être résolues par des spectromètres haute résolution à secteurs, mais beaucoup plus coûteux, exigeant un vide plus poussé et parfois moins sensibles ou bien à l'aide de chambres de collision/réaction.

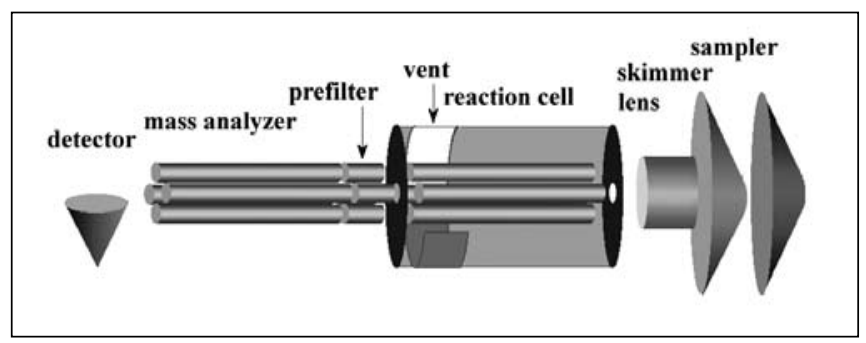

Figure 3 : Schéma du système analytique (interface, optique ionique, DRC, quadrupole, multiplicateur d'électrons) du spectromètre ICP-MS Elan 6100 DRC - Perkin Elmer Sciex.

La partie détection fait classiquement appel à des détecteurs à détection directe des ions comme par exemple les multiplicateurs d'électrons du type channeltron ou à dynodes discrètes. L'évolution actuelle tend à se tourner vers le multiplicateur d'électrons à dynodes discrètes à deux étages, permettant de travailler en mode analogique (pour de fortes concentrations), en mode comptage ou selon les deux modes pour améliorer la dynamique de mesure.

\section{Paramètres de fonctionnement}

Comme pour toute technique analytique, les performances de l'appareil doivent faire l'objet de contrôles quotidiens, lors de la mise en marche de l'appareil et en cours d'analyse. Ces contrôles ont pour principal but l'optimisation du signal. Avant d'effectuer ces réglages et après avoir allumé le plasma, il est impératif d'attendre au moins une demi-heure, temps nécessaire à la stabilisation du plasma. L'optimisation du signal est en effet principalement liée aux paramètres de fonctionnement du plasma :

- puissance du générateurhaute fréquence, classiquement comprise entre 1,2 et $1,4 \mathrm{~kW}$,

- distance spire-interface et positionnement de la torche dans la bobine d'induction, surtout si la torche a été démontée pour un entretien,

- tension de l'optique ionique, qui influe énormément sur la réponse de l'analyte ; pour les masses faibles (Be ou $\mathrm{Mg}$ ) l'optimum se situe à une tension faible (4-6 V) ; il se décale vers les tensions élevées quand le rapport $\mathrm{m} / \mathrm{z}$ augmente $(8-11 \mathrm{~V}$ pour $\mathrm{In}$ ou $\mathrm{Pb})$;

- vitesse et quantité d'aérosol, liées au débit de l'échantillon et au débit des gaz vecteurs ; ce paramètre critique (figure 1) rend indispensable l'utilisation en ICP-MS de débitmètres massiques.

Ces paramètres sont classiquement vérifiés à l'aide de solutions multi-élémentaires et en utilisant les procédures d'optimisation quotidiennes (Daily performance) ou complètes intégrées aux logiciels d'exploitation des appareils.

\section{Difficultés spécifiques liées aux interférences}

Les spectres obtenus en ICP-MS devraient être simples puisqu'ils proviennent en théorie d'ions monoatomiques et de leurs isotopes. La situation est cependant plus complexe et des interférences physico-chimiques (effets de biais de masse, interférences spectrales) surviennent, notamment pour la recherche des éléments traces dans les matrices biologiques chargées. Ces interférences sont classiquement regroupées en interférences nonspectroscopiques et spectroscopiques.

Les interférences non-spectroscopiques sont liées aux effets relatifs :

- au système d'introduction des échantillons. Leurs caractéristiques physiques (viscosité, concentration en acide, concentration en solides dissous) peuvent affecter la nébulisation et le transport de l'aérosol. Il est ainsi préférable de se limiter à des concentrations en sels de l'ordre de $0,1 \%$. Inversement, un ajout modéré (1 à $2 \%)$ d'un alcool aliphatique (C1 à $\mathrm{C} 4)$ peut exalter le signal des éléments As ou Se par augmentation du taux d'ionisation et accroissement de l'efficacité de la nébulisation (12). 
- à l'ionisation de l'échantillon dans le plasma. La présence de concentrations élevées d'éléments facilement ionisables peut entraîner une dépression du signal par déplacement de l'équilibre d'ionisation de l'analyte. Cet effet de matrice est particulièrement net avec les alcalins $(\mathrm{Na}, \mathrm{K}$ ) et les alcalino-terreux (Ca) présents en fortes concentrations dans les fluides biologiques, d'où l'intérêt d'un plasma robuste et d'un réglage optimal du débit de gaz de nébulisation permettant un temps de séjour dans le plasma suffisamment long.

- au transport des ions dans l'interface et dans l'optique ionique. Ces effets physiques de biais de masse diminuent la sensibilité aux ions légers par un défaut de focalisation, le plus souvent lié à un effet chargeespace : les ions de masse lourde et de faible potentiel d'ionisation (Cs, Ba, lanthanides, actinides) affectent le trajet des ions de masse légère, tel Li. Cet effet est néanmoins minimisé par un ajustement continuel de la tension de l'optique ionique des appareils ICP-MS.

De façon plus générale, la résolution de ces interférences non-spectroscopiques peutêtre envisagée par l'utilisation d'un étalon interne de masse et potentiel d'ionisation similaires à ceux de l'analyte, par une dilution de l'échantillon, voire par un étalonnage par ajouts dosés.

Les interférences spectroscopiques, liées à la limite de résolution de l'analyseur (0,5 uma pour un filtre quadripolaire), affectent principalement les éléments de masse atomique inférieure ou égale à 80 et peuvent être réparties en quatre classes (13) :

- les interférences isobariques : elles sont liées à la présence d'un isotope d'un élément différent de l'analyte, mais de rapport $\mathrm{m} / \mathrm{z}$ quasi-identique. Ainsi pour le chrome, ${ }^{50} \mathrm{Cr}^{+},{ }^{50} \mathrm{~V}^{+},{ }^{50} \mathrm{Ti}^{+}$d'une part, ${ }^{54} \mathrm{Cr}^{+}$et ${ }^{54} \mathrm{Fe}^{+} \mathrm{d}$ 'autre part, sont des ions isobares. La résolution de ces interférences isobariques peut se faire, dans la mesure où l'on n'est pas en présence d'éléments mono-isotopiques, par le choix d'un autre isotope non interféré $\left({ }^{52} \mathrm{Cr}^{+}\right.$ou $\left.{ }^{53} \mathrm{Cr}^{+}\right)$ou par l'utilisation d'équations de correction tenant compte des intensités et des abondances des nuclides interférés. Une autre possibilité consiste à travailler en optimisant le plasma dans des conditions beaucoup moins énergétiques ( « plasma froid» d'une puissance de l'ordre de $800 \mathrm{~W}$ ), de manière à limiter l'ionisation aux seuls éléments ayant un potentiel d'ionisation inférieur à $8 \mathrm{eV}$; il est ainsi possible de supprimer l'interférence isobarique entre ${ }^{40} \mathrm{Ca}^{+}$et ${ }^{40} \mathrm{Ar}^{+}$, dans la mesure où l'utilisation de l'ion ${ }^{44} \mathrm{Ca}^{+} \mathrm{n}$ 'aurait pu être retenue. Mais ce mode opératoire peut entraîner une perte de sensibilité, notamment pour les matrices chargées.

- les interférences dues aux ions doublement chargés sont liées à l'existence d'éléments dont la somme des énergies de première et de deuxième ionisation est inférieure à 15,76 eV. C'est le cas du baryum dont le rapport $\mathrm{m} / \mathrm{z}$ peut être divisé par deux du fait de cette double ionisation (interférence entre ${ }^{138} \mathrm{Ba}^{++}$et ${ }^{69} \mathrm{Ga}^{+}$), d'où l'intérêt de l'optimisation du débit de gaz de nébulisation en fonction du rapport $\mathrm{Ba}^{++} / \mathrm{Ba}^{+}$(figure 1). - le recouvrement de pics : il se produit s'il existe un isotope de masse voisine de l'analyte, mais dont la concentration importante ne peut être totalement éliminée de par l'insuffisance de la résolution. Il conviendra donc d'apporter une attention particulière aux éléments, notamment alcalins et alcalinoterreux, particulièrement abondants dans les matrices biologiques.

- les interférences polyatomiques : les ions polyatomiques sont dus, soit à la dissociation incomplète de l'échantillon dans le plasma, soit à la recombinaison au niveau de l'interface d'ions monoatomiques issus du plasma lui-même, de la matrice ou des acides utilisés lors de la préparation de l'échantillon. Les plus communs (tableau II) sont des argures (ArX), des nitrures (NX), des oxydes (XO), des hydroxydes $(\mathrm{XOH})$, des hydrures $(\mathrm{HX})$ et des dimères $\left(\mathrm{X}^{2}\right)$ où $\mathrm{X}$ est n'importe quel élément. Bien que des listes détaillées de ces espèces moléculaires aient été publiées (11), elles restent néanmoins une des principales limitations de la technique ICP-MS.

Tableau II : Principales interférences polyatomiques gênantes pour les analyses en biologie clinique.

\begin{tabular}{|c|c|}
\hline Éléments & Interfaces polyatomiques majeures \\
\hline${ }^{27} \mathrm{Al}^{+}$ & ${ }^{13} \mathrm{C}^{14} \mathrm{~N}^{+},{ }^{12} \mathrm{C}^{15} \mathrm{~N}^{+}$ \\
\hline${ }^{28} \mathrm{Si}^{+}$ & ${ }^{14} \mathrm{~N}_{2}^{+},{ }^{12} \mathrm{C}^{16} \mathrm{O}^{+}$ \\
\hline${ }^{32} \mathrm{~S}^{+}$ & ${ }^{16} \mathrm{O}_{2}^{+}$ \\
\hline${ }^{51} \mathrm{~V}^{+}$ & ${ }^{35} \mathrm{Cl}^{16} \mathrm{O}^{+},{ }^{37} \mathrm{Cl}^{14} \mathrm{~N}^{+}$ \\
\hline${ }^{52} \mathrm{Cr}^{+}$ & ${ }^{40} \mathrm{Ar}^{12} \mathrm{C}^{+},{ }^{40} \mathrm{Ca}^{12} \mathrm{C}^{+},{ }^{35} \mathrm{Cl}^{16} \mathrm{O}^{1} \mathrm{H}^{+}$ \\
\hline${ }^{56} \mathrm{Fe}^{+}$ & ${ }^{40} \mathrm{Ar}^{16} \mathrm{O}^{+},{ }^{40} \mathrm{Ca}^{16} \mathrm{O}^{+}$ \\
\hline${ }^{59} \mathrm{Co}^{+}$ & ${ }^{24} \mathrm{Mg}^{35} \mathrm{Cl}^{+}$ \\
\hline${ }^{63} \mathrm{Cu}^{+}$ & ${ }^{40} \mathrm{Ar}^{23} \mathrm{Na}^{+},{ }^{31} \mathrm{P}^{16} \mathrm{O}_{2}^{+}$ \\
\hline${ }^{64} \mathrm{Zn}^{+}$ & ${ }^{40} \mathrm{Ar}^{24} \mathrm{Mg}^{+},{ }^{32} \mathrm{~S}^{16} \mathrm{O}_{2}^{+}$ \\
\hline${ }^{75} \mathrm{As}^{+}$ & ${ }^{40} \mathrm{Ar}^{35} \mathrm{Cl}^{+},{ }^{40} \mathrm{Ca}^{35} \mathrm{Cl}^{+}$ \\
\hline${ }^{80} \mathrm{Se}^{+}$ & ${ }^{40} \mathrm{Ar}_{2}^{+},{ }^{40} \mathrm{Ar}^{40} \mathrm{Ca}^{+}$ \\
\hline
\end{tabular}

La levée de ces interférences polyatomiques peut être tentée à l'aide d'équations de correction ou par l'utilisation du plasma froid ou de la haute résolution. Une alternative innovante est apparue dans les années 2000 avec les dispositifs de collisions/réactions $(14,15)$. Ces dispositifs sont constitués d'une enceinte contenant un premier multipole, placé en amont de l'analyseur quadripolaire. Un gaz, réactif $\left(\mathrm{NH}_{3}, \mathrm{CH}_{4}\right)$ ou non $(\mathrm{He})$, est introduit dans cette enceinte à un débit optimisé et peut provoquer sur l'ion polyatomique interférant ou 
sur l'analyte lui-même des réactions de :

- Transfert de charge :

$$
\mathrm{ArO}^{+}+\mathrm{NH}_{3} \rightarrow \mathrm{ArO}+\mathrm{NH}_{3}^{+}
$$

- Transfert d'atome d'hydrogène

$$
\mathrm{Ar}_{2}^{+}+\mathrm{NH}_{3} \rightarrow \mathrm{Ar}_{2} \mathrm{H}^{+}+\mathrm{NH}_{2}
$$

- Transfert d'atome d'oxygène

$$
\mathrm{As}^{+}+\mathrm{N}_{2} \mathrm{O} \rightarrow \mathrm{AsO}^{+}+\mathrm{N}_{2}
$$

- Condensation

$$
\mathrm{Se}^{+}+\mathrm{O}_{2} \rightarrow \mathrm{SeO}^{+}+\mathrm{O}
$$

Ces chambres de réaction dynamique $\left(\mathrm{DRC}^{\circledast}\right.$ pour Dynamic Reaction Cell) ou cellules de collision $\left(\mathrm{CCT}^{\circledR}\right)$ éliminent effectivement nombre d'interférences spectrales, mais peuvent également en générer de nouvelles, d'où l'intérêt de filtrer les ions générés, en fonction des systèmes, par leurs masses ou leurs énergies cinétiques. Il n'en demeure pas moins que ces dispositifs prometteurs sont plus efficaces que les plasmas froids et permettent même un gain en sensibilité.

\section{Applications en biologie médicale}

L'étape pré-analytique : La sensibilité et les limites de détection accessibles en ICP-MS réservent plutôt cette technique à la détermination des oligoéléments, des éléments trace $(0,1-100 \mu \mathrm{g} / \mathrm{L})$ et ultra-trace $(<0,1 \mu \mathrm{g} / \mathrm{L})$. Dans ce contexte, un préalable indispensable à l'utilisation d'un ICP-MS en biologie clinique consiste à vérifier si le laboratoire est prêt à accueillir l'appareil dans des conditions d'utilisation et de stabilité optimales (17). Sans vouloir être exhaustif, une attention toute particulière doit être apportée à :

- la maîtrise des contaminations, depuis le prélèvement de l'échantillon jusqu'au terme de son analyse. Ainsi le travail en salle propre (classe 100000 ou 10000 ) est fortement recommandé, notamment pour la détermination aux concentrations physiologiques d'éléments trace ubiquitaires comme l'aluminium. Le GBEA et la norme NF EN ISO/CEI 17025 (septembre 2005) insistent particulièrement sur ces recommandations.

- la climatisation et la ventilation du local. Une extraction régulière des calories (500 à 1000 Joules/s) issues de la torche, du générateur haute fréquence et des pompes nécessite par exemple un débit global d'évacuation gazeuse de plus de $200 \mathrm{~L} / \mathrm{s}$ pour un appareil Elan 6100 DRC, chiffre à mettre en parallèle avec le problème précédemment évoqué de l'aérocontamination.

Un autre paramètre dont la maîtrise est d'autant plus importante que l'on dose des éléments trace est celui du prélèvement (17). A côté du choix de la matrice biologique, classiquement le sang ou l'urine, même si d'autres milieux présentent un intérêt certain (18), il existe un risque réel de contamination du prélèvement lié :

- à la désinfection cutanée (mercure et antiseptiques mercuriels, manganèse et Dakin),

- à l'utilisation d'aiguilles métalliques (chrome, nickel, ...) d'où le recours aux aiguilles siliconées voire aux cathéters en téflon ${ }^{\circledR}$,

- au tube de prélèvement : proscrire les tubes avec séparateur (activateur de coagulation), être vigilant pour les tubes en verre ordinaire, privilégier les tubes spéciaux pour éléments trace et les tubes plastique et dans tous les cas, envisager la possibilité de contaminations métalliques liées au bouchon $(\mathrm{Zn}, \mathrm{Pb}$, $\mathrm{Al}, .$.$) ou à l'anticoagulant.$

La préparation proprement dite des échantillons biologiques pour une analyse en ICP-MS nécessite une dilution de façon à respecter les conditions de salinité et les niveaux de concentration en analyte compatibles avec la technique. A partir d'un volume d'échantillon de quelques centaines de microlitres, le facteur de dilution classiquement appliqué varie de $1 / 5^{\text {ème }}$ à $1 / 10^{\text {ème }}$ pour les matrices peu chargées (urines, plasma) pour atteindre $1 / 20^{\text {ème }}$ pour le sang total. Il peut être fait appel à une simple dilution, le plus souvent en milieu acide par $\mathrm{HNO}_{3}$ 《ultra-pur » (concentration 1 à $2 \%$ ) $(3,19,20)$ ou en milieu basique $\left(\mathrm{NH}_{4} \mathrm{OH}\right.$ à $\left.0,5 \%\right)$ $(21,22)$; en effet l'ammoniaque favorise l'hémolyse et la dissolution des protéines et peut minimiser la formation des interférences polyatomiques type $\mathrm{ArCl}^{+}$par capture des ions chlorure, relativement abondants dans les matrices biologiques (10). L'utilisation d'un digesteur micro-ondes peut s'avérer intéressante, dans la mesure où l'on a pu s'affranchir des effets mémoire (24). Enfin, la solution de dilution peut également comporter :

- un chélateur $(0,1 \mathrm{~g} / \mathrm{L}$ d'EDTA) pour maintenir les éléments volatils dans la solution et éviter un effet mémoire,

- un alcool (4,5 \%o de n-butanol) pour améliorer l'ionisation de certains éléments (12),

- un tensio-actif ( $1 \%$ de Triton X 100) pour favoriser la nébulisation et empêcher l'adhésion de l'échantillon aux parois du nébuliseur,

- l'ajout d'un ou plusieurs étalons internes.

Quelques domaines d'application en biologie clinique: Dans la famille des métaux alcalins, peu d'éléments trace nécessitent le recours à l'ICP-MS pour les analyses de biologie clinique. On peut citer de façon anecdotique les cas du rubidium (24) ou du césium (25) et s'intéresser plus particulièrement au cas du lithium. Les sels de lithium sont effectivement utilisés dans le traitement de la psychose maniaco-dépressive avec la particularité d'une faible marge thérapeutique et d'une grande variabilité inter-individuelle de leur pharmacocinétique. Les concentrations plasmatiques 
usuelles vont de 3,5 à $8,3 \mathrm{mg} / \mathrm{L}(0,5$ à $1,2 \mathrm{mmol} / \mathrm{L})$ pour une concentration toxique à partir de $14 \mathrm{mg} / \mathrm{L}(2 \mathrm{mmol} / \mathrm{L})$ et sont classiquement accessibles par spectrométrie d'absorption atomique (26). Néanmoins, la technique ICP-MS gagne à être utilisée pour la détermination des valeurs physiologiques, en dehors de tout contexte thérapeutique, avec des limites de quantification inférieures au ppb (20), éventuellement abaissables au ppt en travaillant en plasma froid, ce que le faible potentiel d'ionisation de ${ }^{7} \mathrm{Li}(5,39 \mathrm{eV})$ permet (27).

Pour les alcalino-terreux, leur intérêt en biologie clinique est principalement centré sur les éléments majeurs que sont le magnésium et le calcium, dont le dosage est réalisé par d'autres techniques automatisées. Le cas échéant, un dosage du calcium nécessiterait de choisir l'isotope ${ }^{44} \mathrm{Ca}$ (abondance de 2,06\%) pour éviter le recouvrement isobarique entre ${ }^{40} \mathrm{Ca}^{+} \mathrm{et}^{40} \mathrm{Ar}^{+}$. Les autres éléments de cette famille sont plutôt investigués dans le cadre de la surveillance des expositions professionnelles ou environnementales pour le béryllium (28) ou dans un cadre médico-légal de diagnostic de noyade pour le strontium (29), sans poser de problèmes analytiques particuliers dans notre expérience.

Le dosage des éléments trace et ultra-trace prend toute son importance en biologie médicale pour la famille des métaux. En excluant les domaines de la médecine légale et de la toxicologie professionnelle traités par ailleurs, on peut citer, sans faire une liste fastidieuse, des exemples de métaux d'intérêt clinique dont le dosage gagne à être effectué par ICP-MS : le cas du mercure avec la problématique des amalgames dentaires, du plomb avec la surveillance de la plombémie de l'enfant mineur, du platine et du cuivre libres dans le cadre de suivis thérapeutiques (traitements anticancéreux, maladie de Wilson) ou d'autres métaux (V, Cr, Co, Mo, W) pour les patients porteurs de prothèses métalliques. Avant d'aborder ces exemples, il peut être opportun de signaler ici que certains métaux «absents » ou plutôt présents à l'état d'ultra-traces dans les milieux biologiques, pourront être choisis comme étalons internes. En effet, les principaux critères de sélection d'un étalon interne en ICP-MS sont son énergie d'ionisation et son rapport $\mathrm{m} / z$ proches de ceux de l'élément à doser, son absence dans l'échantillon à analyser et la non-création d'interférences spectrales. Ces spécifications nous ont par exemple conduit vers le choix comme étalons internes des éléments tels que ${ }^{45} \mathrm{Sc},{ }^{89} \mathrm{Y},{ }^{115} \mathrm{In},{ }^{187} \mathrm{Rh}$, notamment pour le développement d'un screening multi-élémentaire (21).

- Le mercure demeure un sujet d'actualité, par son omniprésence environnementale et domestique (30). Sa toxicité est étroitement liée à sa spéciation et les valeurs usuelles pour des sujets non exposés sont inférieures à $10 \mu \mathrm{g} / \mathrm{L}$ pour la mercuriémie, à $7 \mu \mathrm{g} / \mathrm{L}$ pour la mercuriurie et à $4 \mu \mathrm{g} / \mathrm{L}$ pour le mercure plasmatique. Or c'est justement le plasma qui est la meilleure matrice pour pouvoir apprécier l'imprégnation mercurielle liée à la présence d'amalgames dentaires et il est communément admis une corrélation positive entre le nombre d'amalgames dentaires et le mercure plasmatique : augmentation de $0,02 \mu \mathrm{g} / \mathrm{L}$ par surface unitaire d'amalgame, avec des variations individuelles considérables (31). Dans le cadre de sa mission de surveillance, l'Agence française de sécurité sanitaire des produits de santé a rédigé sur cette problématique un rapport «Le mercure des amalgames dentaires » (32), préconisant entre autres recommandations, la mise en place d'un protocole d'examen multidisciplinaire (médecin toxicologue et odontologiste) destiné aux personnes qui présentent des troubles qu'elles estiment liés à la présence d'amalgames dentaires. Le bilan biologique comporte notamment un dosage plasmatique du mercure que nous avons développé par ICP-MS. L'analyse est faite sur $1 \mathrm{le}^{202} \mathrm{Hg}$ et vérifiée sur l'isotope ${ }^{200} \mathrm{Hg}$, en diluant le plasma au $1 / 20^{\text {ème à }}$ l'aide de la solution précédemment présentée pour le screening (21). Le dosage est réalisé par étalonnage externe, l'effet de matrice ayant été levé par réalisation d'une gamme dans du « sérum synthétique » $(\mathrm{NaCl}$ $0,75 \% ; \mathrm{CaCl}_{2} 0,05 \%$ ) avec le rhénium pour étalon interne. Dans ces conditions, la quantification est possible sur la gamme $0,5-50,0 \mu \mathrm{g} / \mathrm{L}$ (figure 4).

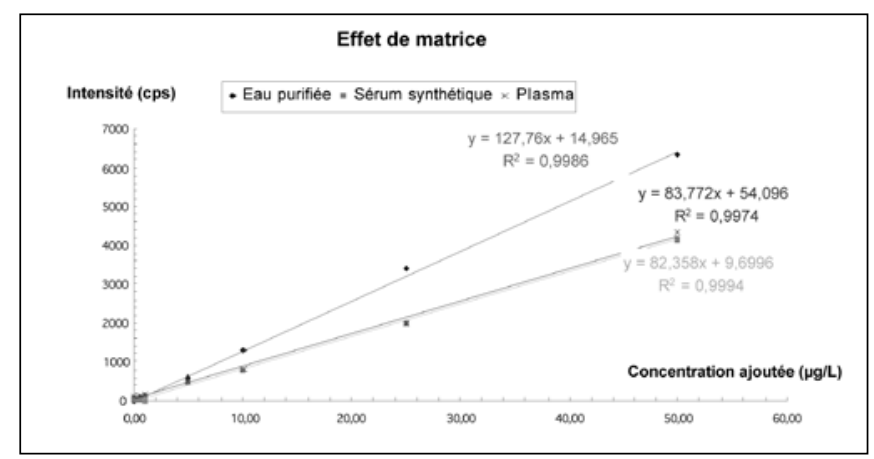

Figure 4: Dosage par ICP-MS du mercure plasmatique. Résolution de l'effet de matrice à l'aide d'un étalonnage externe dans du sérum synthétique.

- La surveillance biologique de l'exposition au plomb concerne essentiellement les personnes professionnellementexposéeset lesenfants vivants dans des conditions d'hygiène douteuses. A la demande de l'Institut de Veille Sanitaire et dans le cadre du système national de surveillance des plombémies, une étude multicentrique a été réalisée par le groupe de travail «Toxiques Industriels » de la SFTA sur l'incertitude de mesure pour les valeurs basses de la plombémie et sur la détermination de la limite de quantification (Ldq) pour le dosage du plomb sanguin (33). Par rapport à la 
spectrométrie d'absorption atomique électrothermique, les résultats, en cours de publication, montrent une meilleure sensibilité de la méthode ICP-MS pour le dosage du plomb sanguin, avec une Ldq calculée dans notre expérience inférieure à $5 \mu \mathrm{g} / \mathrm{L}$ (étalonnage externe dans une matrice synthétique $(\mathrm{NaCl} 0,75 \%$; $\left.\mathrm{CaCl}_{2} 0,05 \%\right)$ - dilution du sang au $1 / 45^{\text {ème }}$ - étalon interne : ${ }^{187} \mathrm{Re}$ ) (34).

- Depuis quelques années, l'augmentation de l'utilisation du platine dans ses applications médicales et industrielles (pots catalytiques,...) entraîne une exposition des personnes, patients ou professionnels. En biologie médicale, le suivi thérapeutique pharmacologique des patients sous traitement anti-néoplasique par les dérivés du platine (cisplatine, carboplatine, oxaliplatine) nécessite un dosage préférentiel de la forme plasmatique libre. En effet, l'aire sous la courbe du platine ultra-filtrable est corrélée linéairement à la toxicité hématologique dose-limitante du médicament (35). Dans ce contexte, comme dans celui des expositions diffuses, le dosage du platine classiquement effectué par spectrométrie d'absorption atomique électrothermique gagne à être réalisé par ICP-MS. Outre le gain en sensibilité, la technique permet également de réaliser une spéciation du platine par couplage avec la chromatographie liquide (HPLC) (36). Ce couplage HPLC-ICP-MS peut même présenter une amélioration par rapport aux performances obtenues par couplage HPLC-MS/MS (37).

- Pour terminer sur des exemples de métaux investigués dans le cadre de la biologie clinique, on peut citer le cas du chrome et de son relargage chez les patients porteurs de prothèses totales de hanche en métal. En effet, les complications à long terme des arthroplasties totales de hanche sont dominées par l'usure des composants prothétiques et la dissémination de particules de chrome, vanadium, titane ou cobalt dans l'organisme (38). Le cas du chrome est d'autant plus intéressant que son isotope le plus abondant ${ }^{52} \mathrm{Cr}$ (abondance 83,76\%) est le siège d'une interférence polyatomique majeure $\left({ }^{40} \mathrm{Ar}^{12} \mathrm{C}\right)$ dont la résolution nécessite l'usage de la DRC. La levée de cette interférence peut être réalisée par injection d'ammoniac dans la $\mathrm{DRC}$, selon la réaction : ${ }^{40} \mathrm{Ar}^{12} \mathrm{C}^{+}+\mathrm{NH}_{3} \rightarrow{ }^{40} \mathrm{Ar}+{ }^{12} \mathrm{C}+\mathrm{NH}_{3}^{+}$

Il est par contre nécessaire d'optimiser les conditions opératoires de la DRC par de multiples essais en jouant, pour différentes surcharges en chrome, sur les paramètres de rejets (Rpq) du filtre quadripolaire ainsi que sur le débit d'ammoniac (figure 5). Ces essais nous ont permis de mettre au point le dosage du chrome dans le sang par ICP-MS (méthode des ajouts dosés ; $\mathrm{Rpq}=0,7 ;$ débit de $\mathrm{NH}_{3}=0,6 \mathrm{~mL} / \mathrm{min} ; \mathrm{Ldq} 1 \mu \mathrm{g} / \mathrm{L}$ ) (16) sans nécessiter l'usage d'un spectromètre haute résolution et en satisfaisant totalement aux contrôles de qualité externes (German External Quality Assessment Scheme-Erlangen).

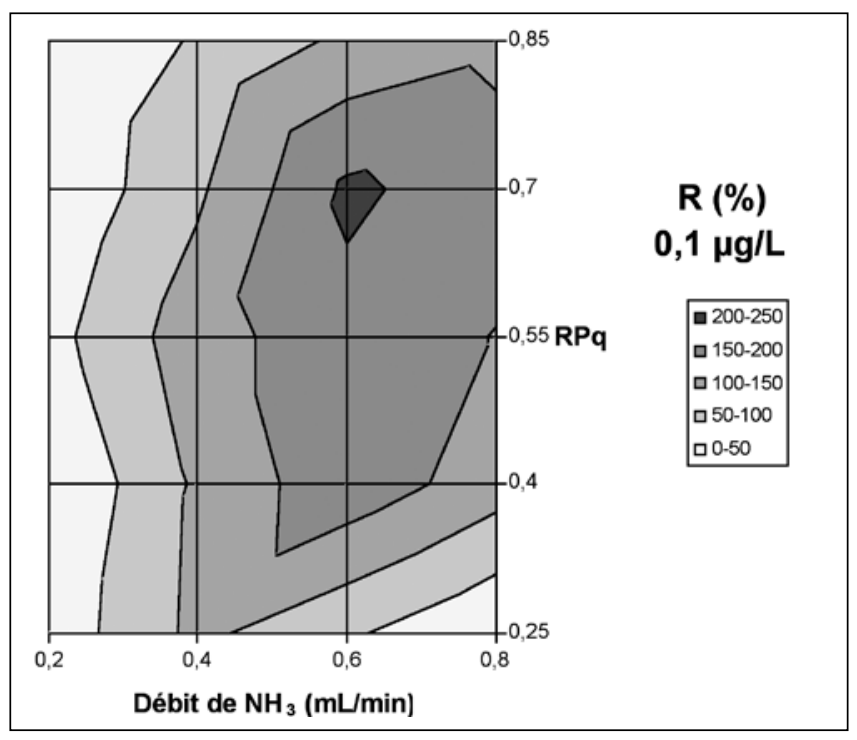

Figure 5 : Dosage du chrome sanguin par ICP-MS-DRC : détermination de la réponse maximale $(R \%)$ pour une surcharge de $0,1 \mu \mathrm{g} / \mathrm{L}$ dans un pool de sang total en fonction de la valeur du paramètre de réjection $q(R P q)$ et du débit d'ammoniac (16).

Ce survol des éléments présentant un intérêt en biologie clinique doit également évoquer des exemples de nonmétaux. On peut aborder le cas de métalloïdes tels que l'arsenic ou le sélénium, pour lesquels la spéciation et l'utilisation de la DRC prennent toute leur importance.

- La toxicité systémique élevée des dérivés inorganiques de l'arsenic contraste avec la très faible toxicité des dérivés organiques (arsénocholine, arsénobétaïne) liés à la consommation de produits de la mer. Dans le cadre de la biologie clinique, l'arsenic $\left(\mathrm{As}_{2} \mathrm{O}_{3}\right)$ a montré son efficacité dans le traitement des leucémies promyélocytaires, mais la tolérance relative du produit administré par voie intraveineuse et la toxicité des différentes espèces arsenicales issues du métabolisme de l'arsenic imposent un suivi thérapeutique réalisé par couplage HPLC-ICP-AES (39) mais qui peut également être envisagé par HPLC-ICP-MS $(40,41)$. Le caractère mono-isotopique de ${ }^{75} \mathrm{As}$ et l'interférence polyatomique majeure ${ }^{40} \mathrm{Ar}^{35} \mathrm{Cl}^{+}$justifierait par contre l'utilisation d'une chambre de collision/réaction ou d'une DRC pour pouvoir réaliser un dosage au rapport $m / z 75$, tout en conservant une sensibilité optimale (42).

- Le sélénium, constituant essentiel de plusieurs enzymes à vocation antioxydante (glutathion peroxydase,...), joue un rôle important dans la protection de l'organisme contre les radicaux libres oxygénés. Il jouerait donc un rôle protecteur au cours des phénomènes physiologiques du vieillissement et du développement de certaines maladies dégénératives. Son dosage 
sérique permet d'évaluer le statut nutritionnel des patients et est d'autant plus important que les symptômes de carence sévères peuvent être caractérisés par des myopathies périphériques, des anémies, voire par une cardiomyopathie nécrosante (maladie de Keshan) évoluant vers une décompensation soudaine et l'arrêt cardiaque. Contrairement à l'arsenic, le sélénium possède 6 isotopes qui sont tous soumis soit à une interférence isobarique, soit à une interférence polyatomique, soit aux deux simultanément. L'isotope le plus abondant ${ }^{80} \mathrm{Se}(49,82 \%)$ présente le même rapport que le dimère ${ }^{40} \mathrm{Ar}^{40} \mathrm{Ar}$. Pour des raisons thermodynamiques de cinétiques réactionnelles, la levée de cette interférence ne peut être réalisée par l'utilisation de la DRC avec l'ammoniac. L'isotope 77 $(7,58 \%)$ est interféré par l'ion polyatomique ${ }^{40} \mathrm{Ar}^{37} \mathrm{Cl}^{+}$ dont la probabilité d'existence est importante dans les liquides biologiques. Ainsi le dosage du sélénium sérique que nous avons développé est-il effectué sur l'isotope ${ }^{82} \mathrm{Se}(9,19 \%)$. L'interférence isobarique imputable au ${ }^{82} \mathrm{Kr}$ est corrigée de façon mathématique par le logiciel et l'isotope ${ }^{77} \mathrm{Se}$ est utilisé comme masse de confirmation avec utilisation de la DRC (10). Dans ces conditions, la limite de détection dans le sérum est de $2 \mu \mathrm{g} / \mathrm{L}$ et la gamme est linéaire jusqu'à $200 \mu \mathrm{g} / \mathrm{L}$. Nous rappelons que les valeurs usuelles sériques vont de 60 à $120 \mu \mathrm{g} / \mathrm{L}$.

Le dernier domaine d'application de l'ICP-MS en biologie clinique qui mérite d'être présenté dans le cadre de cette synthèse est celui du dosage multi-élémentaire dans les milieux biologiques. Nombreuses sont les publications de méthodes de criblage ou « screening » tant dans le cadre des analyses médico-légales que lors d'expositions professionnelles ou environnementales diffuses voire dans le cas de syndromes d'étiologie imprécise $(13,19-23)$. Le biologiste doit pouvoir alors disposer de valeurs usuelles afin de dépister une concentration anormalement élevée ou à l'inverse une carence élémentaire. Le tableau III présente les valeurs usuelles « sang frais » du screening semi-quantitatif développé dans notre laboratoire sur 29 éléments, y compris sur certains éléments dont la détermination nécessite le recours à la DRC. La mise au point a été réalisée par étapes successives (élimination des interférences spectrales pour le vanadium, le chrome, le fer par utilisation de la DRC, étude des effets de matrice, sélection des étalons internes, évaluation des limites de détection et de quantification, étude de la linéarité, de la répétabilité et de l'exactitude). La validité de l'étalonnage a été vérifiée à l'aide de contrôles de qualité internes préparés dans du sang humain lyophilisé (21).
Tableau III : Concentrations usuelles dans le sang total pour les 29 éléments du screening ICP-MS-DRC (valeurs portant sur 52 sujets non exposés).

\begin{tabular}{|l|c|c|c|c|c|}
\hline \multirow{2}{*}{ Éléments } & \multicolumn{5}{|c|}{ Valeurs sang frais $(\boldsymbol{\mu g} / \mathbf{L})$} \\
\cline { 2 - 6 } & Moyenne & Écart-type & Médiane & $\begin{array}{c}\mathbf{5}^{\text {eme }} \\
\text { percentile }\end{array}$ & $\begin{array}{c}\mathbf{9 5} \text { 'me } \\
\text { percentile }\end{array}$ \\
\hline Lithium & 3,65 & 7,35 & 1,10 & 0,40 & 15,74 \\
\hline Béryllium & 0,002 & 0,015 & 0,000 & 0,000 & 0,000 \\
\hline Magnésium & 32552 & 3091 & 32307 & 28151 & 37938 \\
\hline Vanadium & 0,069 & 0,071 & 0,000 & 0,000 & 0,200 \\
\hline Chrome & 0,46 & 0,53 & 0,30 & 0,01 & 1,49 \\
\hline Manganèse & 5,87 & 2,28 & 5,50 & 2,86 & 9,38 \\
\hline Fer & 446299 & 198599 & 377870 & 275890 & 979547 \\
\hline Cobalt & 0,083 & 0,140 & 0,000 & 0,000 & 465 \\
\hline Cuivre & 793 & 119 & 796 & 626 & 960 \\
\hline Zinc & 4922 & 756 & 4865 & 3579 & 5933 \\
\hline Arsenic & 4,00 & 1,48 & 3,55 & 2,59 & 7,70 \\
\hline Sélénium & 91,7 & 13,7 & 92,0 & 71,9 & 114,8 \\
\hline Strontium & 17,9 & 9,4 & 15,0 & 7,3 & 35,4 \\
\hline Molybdène & 0,56 & 0,21 & 0,50 & 0,30 & 0,99 \\
\hline Rhodium & 0,029 & 0,010 & 0,025 & 0,016 & 0,042 \\
\hline Palladium & 4,37 & 3,37 & 4,25 & 0,89 & 12,03 \\
\hline Cadmium & 6,82 & 5,10 & 5,60 & 1,99 & 15,87 \\
\hline Etain & 0,38 & 0,51 & 0,19 & 0,02 & 0,74 \\
\hline Antimoine & 0,39 & 0,24 & 0,30 & 0,30 & 0,70 \\
\hline Baryum & 65,0 & 11,7 & 62,6 & 53,3 & 80,4 \\
\hline Cérium & 3,11 & 0,63 & 3,10 & 2,09 & 4,40 \\
\hline Tungstène & 0,022 & 0,044 & 0,019 & 0,003 & 0,117 \\
\hline Platine & 0,010 & 0,023 & 0,002 & 0,000 & 0,012 \\
\hline Mercure & 2,34 & 1,71 & 2,35 & 0,40 & 5,22 \\
\hline Thallium & 0,021 & 0,007 & 0,021 & 0,013 & 0,030 \\
\hline Plomb & 46,0 & 29,6 & 39,3 & 14,6 & 69,8 \\
\hline Bismuth & 0,003 & 0,004 & 0,003 & 0,000 & 0,007 \\
\hline Thorium & 0,100 & 0,141 & 0,095 & 0,000 & 0,176 \\
\hline Uranium & 0,005 & 0,005 & 0,005 & 0,000 & 0,015 \\
\hline
\end{tabular}

\section{Conclusion}

Les techniques d'ICP-MS appliquées à la biologie clinique doivent et peuvent présenter désormais les qualités suivantes : bonne sensibilité, bonne spécificité, gamme de linéarité étendue. Elles sont utilisables pour effectuer des dosages en série, dans des matrices biologiques chargées et à une cadence analytique élevée. Elles donnent accès aux pléiades isotopiques. Depuis quelques années, l'émergence de solutions innovantes capables de résoudre les principales interférences spectroscopiques (cellules de réaction, ...) ouvre de nouvelles possibilités, jusque là seulement accessibles aux appareils à haute résolution. Ces avancées technologiques permettent de doser plus de cinquante 
éléments, aussi bien dans le domaine des troubles nutritionnels (carences ou excès en oligoéléments), que dans celui des expositions environnementales ou professionnelles (métaux divers, sélénium) ou du suivi thérapeutique pharmacologique (lithium plasmatique, platine et cuivre sériques libres) en vue d'adaptations de posologies.

\section{Références}

1. Houk R.S., Fassel V.A., Flesh G.D., Svec H.J., Gray A.L., Taylor C.E. Inductively coupled argon plasma as an ion source for mass spectrometric determination of trace elements. Anal. Chem. 1980 ; 52 : 2283-89.

2. Patriarca M. The contribution of inductively coupled plasma mass spectrometry to biomedical research. Microchem. J. 1996 ; 54 : 262-71.

3. Labat L., Dehon B., Dhorne C., Lhermitte M. Dosage de métaux par ICP-MS dans différents milieux biologiques. Ann. Toxicol. Anal. 2003 ; 15 : 281-6.

4. Goullé J.P., Mahieu L., Lainé G., Lacroix C., Clarot F., Vaz E., Proust B. Intérêt de la torche à plasma en biologie clinique. Quelles applications en toxicologie ? Ann. Pharm. Fr. 2004 ; 62 : 291-96.

5. Pénicaut B., Bonnefoy C., Moesch C., Lachâtre G. Spectrométrie de masse à plasma couplé par induction (ICP-MS). Potentialités en analyse et en biologie. Ann. Pharm. Fr. 2006 ; 64 : 312-27.

6. Mermet J.M., Poussel E. Couplage plasma induit par haute fréquence - spectrométrie de masse. In : Techniques de l'ingénieur, traité Analyse et Caractérisation. Editions techniques, Paris 1999 ; P2720 : 1-11.

7. Ducros V., Ruffieux D., Leloup C., Dall'ava D. Spectrométrie de masse à plasma induit par haute fréquence. In : Baruthio F. Assurance de qualité de l'analyse des éléments trace en biologie. Lavoisier TEC et DOC - Editions Médicales Internationales, Paris 1997 ; 11 : 167-82.

8. Montaser A. Inductively coupled plasma mass spectrometry. WILEY-VCH, New York 1998 ; 964 p.

9. Holland J.G., Bandura D.R. Plasma source mass spectrometry : Current trends and future developments. RSC Publishing, Cambridge 2005 ; 356 p.

10. Bonnefoy C. Dosage d'éléments trace dans les liquides biologiques par spectrométrie de masse inorganique couplée à un plasma induit par haute fréquence (ICPMS) : mise en œuvre d'une chambre de réaction dynamique (DRC). Thèse de Doctorat, Université Claude Bernard Lyon I, 2005 ; 209 p.

11. May T.W., Wiedmeyer R.H. A table of polyatomic interferences in ICP-MS. Atomic Spectroscopy 1998 ; $19: 150-55$.

12. Allain P., Jaunault L., Mauras Y., Mermet J.M., Delaporte T. Signal enhancement of elements due to the presence of carbon-containing compounds in inductively coupled plasma mass spectroscopy. Anal. Chem. 1991 ; 63 : 1497-8.
13. Hsiung C.S.,Andrade J.D., Costa R., Ash K.O. Minimizing interferences in the quantitative multi-element analysis of trace elements in biological fluids by inductively coupled plasma mass spectrometry. Clin. Chem. 1997 ; $43: 2303-11$.

14. Tanner S.D., Baranov V.L. Theory, design and operation of a dynamic reaction cell for ICP-MS. Atomic Spectroscopy $1999 ; 20: 45-52$.

15. Tanner S.D., Baranov V.L., Bandura D.R. Reaction cells and collision for ICP-MS (review). Spectrochimica Acta part B $2002 ; 57: 1361-452$.

16. Bonnefoy C., Menudier A., Moesch C., Lachâtre G., Mermet J.M. Determination of chromium in whole blood by DRC-ICP-MS: spectral and non-spectral interferences. Anal. Bioanal. Chem. 2005 ; 383 : 167-73.

17. Baruthio F. (Coordonnateur). Assurance de qualité de l'analyse des éléments trace en biologie. Lavoisier TEC et DOC - Editions Médicales Internationales, Paris 1997 ; $216 \mathrm{p}$.

18. Goullé J.P. Metals In : Kintz P. Analytical and practical aspects of drug testing in hair. CRC Press, New York $2007 ; 16: 343-369$.

19. De Boer J.L.M., Ritsema R., Piso S., Van Staden H., Van den Beld W. Practical and quality-control aspects of multi-element analysis with quadrupole ICP-MS with special attention to urine and whole blood. Anal. Bioanal. Chem. 2004 ; 379 : 872-880.

20. Goullé J.P., Mahieu L., Castermant J., Neveu N., Bonneau L., Lainé G., Bouige D., Lacroix C. Metal and metalloid multi-elementary ICP-MS validation in whole blood, plasma, urine and hair. References values. Forensic Sci. Int. $2005 ; 153: 39-44$.

21. Bonnefoy C., Menudier A., Moesch C., Mermet J.M., Lachâtre G. Screening des éléments inorganiques dans le sang total par spectrométrie de masse à plasma induit par haute fréquence (ICP-MS) associée à une chambre de réaction dynamique (CRD). XI ${ }^{\text {ème }}$ Congrès annuel de la Société Française de Toxicologie Analytique, Dinard, 11-13 juin 2003. Ann. Toxicol. Anal., 2003, XV, 161.

22. Heitland P., Köster H.D. Biomonitoring of 37 trace elements in blood samples from inhabitants of northern Germany by ICP-MS. J. Trace Elem. Med. Biol. 2006 ; $20: 253-62$.

23. Rodushkin I., Ödman F., Olofsson R., Axelsson M. Determination of 60 elements in whole blood by sector field inductively coupled plasma mass spectrometry. J. Anal. At. Spectrom. $2000 ; 15$ : 937-44.

24. Domej W., Krachler M., Goessler W., Maier A., Irgolic K.J.,Lang J.K. Concentrations of copper, zinc, manganese, rubidium and magnesium in thoracic empyemata and corresponding sera. Biol. Trace Elem. Res. 2000 ; 78 : 53-66.

25. Alimonti A., Forte G., Spezia S., Gatti A., Mincione G., Ronchi A., Bavazzano P., Bocca B., Minoia C. Uncertainty of inductively coupled plasma mass spectrometry based measurements : an application to the analysis of urinary barium, cesium, antimony and tungsten. Rapid Commun. Mass Spectrom. $2005 ; 19$ : 3131-38.

26. Gonzalez S., Navarro M., Lopez H., Lopez M.C., Perez V. Determination of lithium in human serum by electrothermal atomic absorption spectrometry. J. AOAC. Int. $2000 ; 83: 377-81$. 
27. Bianchi F., Careri M., Maffini M., Mangia A., Mucchino C. Use of experimental design for optimisation of the cold plasma ICP-MS determination of lithium, aluminium and iron in soft drinks and alcoholic beverages. Rapid Commun. Mass Spectrom. 2003 ; 17 : 251-56.

28. Apostoli P., Schaller K.H. Urinary beryllium: a suitable tool for assessing occupational and environmental beryllium exposure? Int. Arch. Occup. Environ. Health $2001 ; 74$ : 162-6.

29. Azparren J.E., Fernandez-Rodriguez A., Vallejo G. Diagnosing death by drowning in fresh water using blood strontium as an indicator. Forensic Sci. Int. 2003 ; 137 : 55-59.

30. Conter S.A., Buchanan L.H. Mercury exposure in children : a review. Toxicol. Applied Pharmacol. 2004 ; 198 : 209-30.

31. Bergdahl I.A., Schütz A., Ahlqwist M., Bengtsson C., Lapidus L., Lissner L., Hultén B. Methylmercury and inorganic mercury in serum - Correlation to fish consumption and dental amalgam in a cohort of women born in 1922. Environ. Research 1998 ; 77 : 20-24.

32. Affsaps. Le mercure dans les amalgames dentaires. Octobre 2005, 103 p (www.afssaps.sante.fr)

33. Olichon D., Labat L., Poupon J., Bost M., Haufroid V., Moesch C., Nicolas A., Furet Y, Goullé J.P., Guillard O., Le Bouil A., Pineau A. Approche analytique de la détermination de la limite de quantification pour le dosage du plomb sanguin : étude multicentrique. Ann. Toxicol. Anal., 2006, 3, 166-7.

34. Bonnefoy C., Menudier A., Moesch C., Lachâtre G., Mermet J.M. Validation of the determination of lead in whole blood by ICP-MS. J. Anal. At. Spectrom. 2002 ; 17 : 1161-65.

35. Canal P., Chatelut E., Rousseau A., Marquet P., Le Guellec C. Suivi thérapeutique du carboplatine. In : Marquet P. Suivi thérapeutique pharmacologique. Elsevier, Paris 2004 : 249-60.
36. Bell D.N., Liu J.J., Tingle M.D., McKeage M.J. Specific determination of intact cisplatin and monohydrated cisplatin in human plasma and culture medium ultrafiltrates using HPLC on-line with inductively coupled plasma mass spectrometry. J. Chromatogr. B, 2006 ; 837 : 29-34.

37. Smith C.J., Wilson I.D., Abou-Shakra F., Payne R., Parry T.C., Sinclair P., Roberts D.W. A comparison of the quantitative methods for the analysis of the platinumcontaining anticancer drug [cis-[amminedichloro (2-methylpyridine)]platinum II] (ZD0473) by HPLC coupled to either a triple quadrupole mass spectrometer or an inductively coupled plasma mass spectrometer. Anal. Chem., 2003 ; 75 : 1463-9.

38. Savarino L., Granchi D., Ciapetti G., Cenni E., Greco M., Rotini R., Veronesi C.A., Baldini N., Giunti A. Ion release in stable hip arthroplasties using metal-on-metal articulating surfaces : A comparison between short- and medium-term results. J. Biomed. Mater. Res. A, 2003 ; $66: 450-6$.

39. Do B., Alet P., Pradeau D., Poupon J., Guilley-Gaillot M., Guyon F. On-line reversed-phase liquid chromatography hydride generation emission spectrometry : speciation of arsenic in urine of patients intravenously treated with $\mathrm{As}_{2} \mathrm{O}_{3}$. J. Chromatogr. B Biomed. Sci. Appl., 2000 ; $740: 179-86$.

40. Mandal B.K., Ogra Y., Anzai K., Suzuki K.T. Speciation of arsenic in biological samples. Toxicol. Appl. Pharmacol., 2004 ; 198 : 307-318.

41. Morton J.and Mason H. Speciation of arsenic compounds in urine from occupationally unexposed and exposed persons in the U.K. using a routine LC-ICP-MS method. J. Anal. Toxicol., $2006 ; 30: 293-301$.

42. Darrouzes J., Bueno M., Simon S., Pannier F., PotinGautier M. Optimisation d'un ICPMS à cellule de collision/réaction pour l'analyse de l'arsenic et du sélénium dans les eaux naturelles. Eur. J. Water Quality, $2006 ; 37: 65-74$. 